\title{
HUNDIMIENTOS ACTIVOS CONTROLADOS POR ESTRUCTURAS DEL BASAMENTO EN LA CUENCA MARAÑON (NORESTE DEL PERU) (*)
}

J.F. Dumont ${ }^{* *}$

F. García ${ }^{* * *}$

\section{RESUMEN}

La cuenca del Marañón está localizada entre el Escudo brasilero y las estribaciones orientales de los Andes. La parte del sur de la cuenca, denominada depresión U camara, comprende grandes áreas inundadas y meandros, con bordes más 0 menos rectos y Forma casi geométrica. El área de inundación "Punga', es un ejemplo histórico de tectónica relacionada con hundimientos (subsidencia); grandes áreas inundables recubren bloques estructurales limitados por fallas y levantados o cabalgados sobre estructuras relacionadas con la reactivación de las fallas del basamento; las implicaciones para el contexto de la geodinámica regional son brevemente discutidas.

\section{ABSTRACT}

The $M$ arañon basin is located between the Brazilian shield and the Andean foothilis The shouthern part of the basin, called U camara depression, comprises large swamps and lakes, with more or less straight edges and nearly geometrical shapes. The Pung swap is an historical example of tectonic related subsidence. Large swamps overlay structural block limited by faults, and uplifted or pulled dowm basement structures. The main trend of the swamps is related to the reactivation of basement faults. The implications for the regional geodynamic contex are briefly discussed.

Palabras Claves: Cuenca del M arañón, Perú, Tectónica, Subsidencia, Estructura del Basamento, Geodinámica.

L ands Subsidence (Proceedings of the Four International Symposium on Land Subsidence, M ay 1991).

IA HS Publ. Nํ200, 1991

Convenio ORSTOM-IIAP, Apartado 18-1209 Lima-Perú. Dirección actual ORSTOM CP 9214, La Paz Bolivia.

ORSTOM - IGP 


\section{INTRODUCCION}

La cuenca del Marañón, ubicada en el noreste del Perú, está localizada entre el Escudo brasilero, al este, representado por las tierras altas de Iquitos y las estribaciones de los Andes, al oeste. (Fig. 1). El área de drenaje de la cuenca del M arañón comprende el gran pie de monte de Ios Andes orientales, que se extiende exactamente debajo del Ecuador hasta cerca del lago Titicaca, sobre los 140 de Latitud. Esto proporciona gran cantidad de agua provenientes de los Andes y concentrada en dos grandes ríos EI U cayali y el Marañón. El clima tropical húmedo genera abundantes precipitaciones sobre las áreas de las tierras bajas de la cuenca, drenada por una densa red de ríos; en el contexto del activo hundimiento (subsidencia), ésta región parece ser un buen ejemplo para el estudio de modelos de tierras húmedas y fluviales en relación a neotectónismos.

M ientras que los estudios neotectónicos en áreas de levantamientos están bien documentados y basados en varios métodos básicos (A nálisis de formas de tierras, escarpas de falla y mediciones de planos de fallas), las áreas de subsidencia están pobremente documentadas, porque los métodos actuales neotectónicos no lo hacen aplicable. Los estudios neotectónicos en la cuenca amazónica empiezan probablemente con Stemberg (1950 -1955). En las regiones del oeste amazónico, Ruegg 1952, menciona deformaciones de pliegues en los últimos depósitos lacustres del Terciario, atribuidos a la fase tectónica del Plio-Cuaternario; últimamente, Iriondo y Sugio (1981), hacen notar el relativo efecto de basculamiento y subsidencia en los valles de los ríos de la amazonia. En la cuenca del Beni (Bolivia), cuya posición es similar a esta del río Marañón, Allenbry (1988), señala que el alineamiento de forma rectangular de los lagos se presenta como controlado por un modelo de fractura octogonal, propagado hacia arriba del basamento granítico infrayaciente, aunque la carencia de datos de precisión estructural para el basamento haga que la hipótesis de Allenby resulte especulativa. El ejemplo considerado aquí está basado en estrechas relaciones entre modelos de superficie observados en imágenes $L$ andsat y SLAR e interpretaciones estructurales de basamento de datos sísmicos (Laurent y Pardo 1975; Laurent 1985) se presenta aquí nuevos datos sobre la subsidencia de la región, efectuados en base de estudios de campo.

\section{ANTECEDENTES GEOLOGICOS}

La cuenca del Marañón (Fig. 1) ha sido desarrollada principalmente durante el Cenozoico (Terciario); de acuerdo con Laurent y Pardo (1975) y Laurente (1985), Ios depósitos del Triásico superior al Jurásico existen solamente en la parte occidental de la cuenca y los depósitos Cretáceos (A ptiano - M aestriachiano) traslapan hacia el este, sobre el Paleozoico y el basamento cristalino. El espesor de los sedimentos post- Jurásicos se eleva a 5,000 m. en la parte central de la cuenca (Sanz, 1974), pero las más notorias características tectónicas del basamento son última fase Kimmeridgiana. Estos rangos son representados por aislados horsts del basamento cristalino o por delgadas capas sedimentarias, recubiertas por depósitos Cretáceos (de norte a sur: levatamientos Concordia, Samiria, Santa Elena y Santa Lucía). Fallas transcurrentes son reportadas para la última tectónica H ercyniana. 


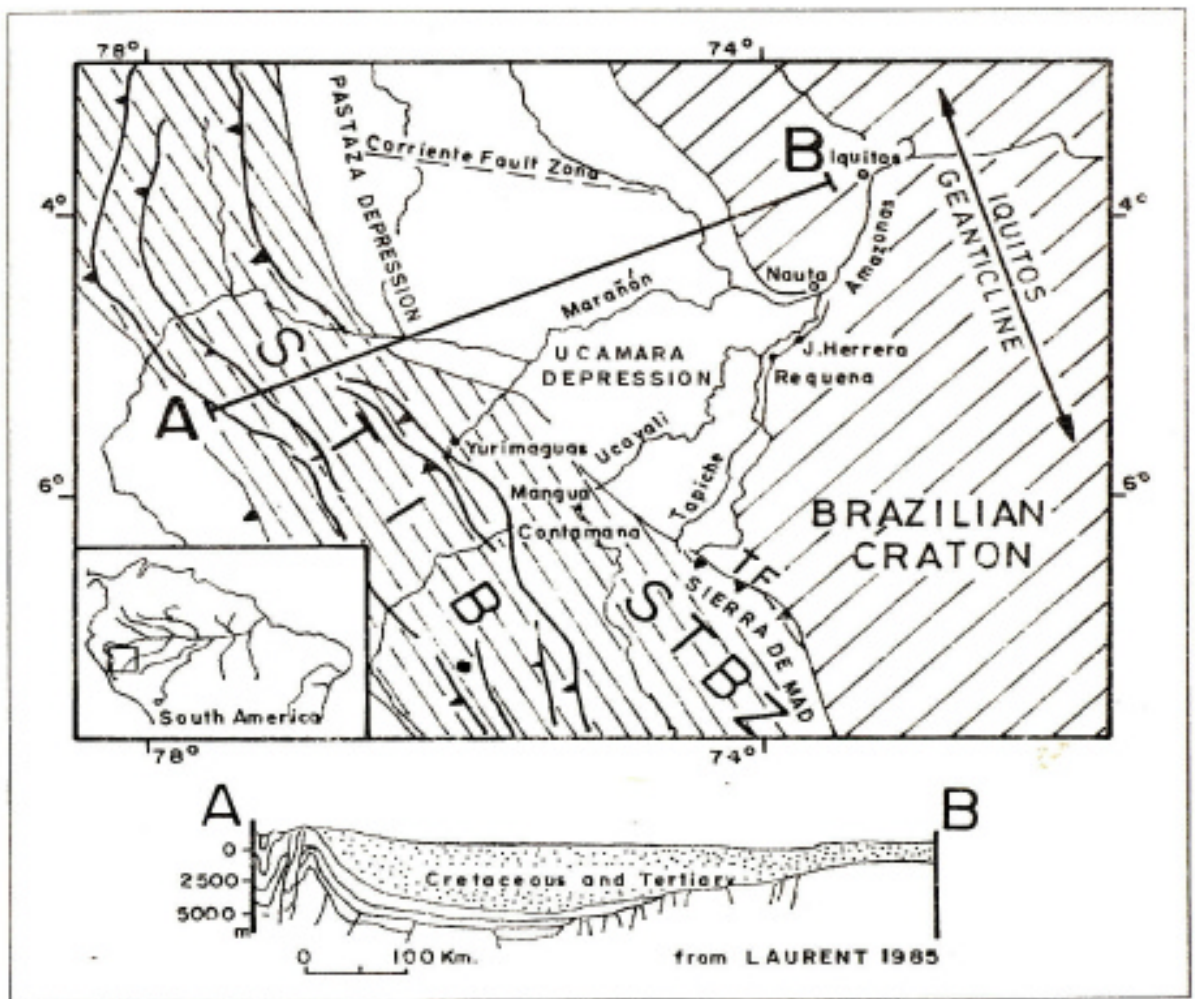

Fig. 1 Esquema estructural de la cuenca del Marañón y áreas circundantes.

STFB : Sobreescurrimientos y cinturón de pliegues subandino

STBZ : Zona basculada subandina.

A-B : Sección cruzada de la cuenca del marañón

T-F : Falla Tapiche

La subsidencia de la cuenca fue acompañada por tendencias positivas en el geoanticlinal Iquitos; durante el último Terciario, la subsidencia fue acelerada sobre el conjunto del área y sobre los depósitos del Mio-Plioceno extendidos sobre el geoanticlinal. Durante el Mesozoico y Cenozoico, la profunda parte delantera de la cuenca andina fue mucho más extendida que ahora, longitudinalmente a lo largo de la Cordillera de los Andes del Perú y también; lateralmente sobre las presentes estribaciones. La cuenca fue reducida a la presente extensión como un resultado del último tectonismo en el Terciario y temprano Quaternario en el área de sobre escurrimiento y cinturón de pliegues subandino. (Ham. y Herrera, 1963; Pardo 1982; Megard, 1984), así como en las tierras del frente subandino del Perú central, que fue completamente levantado como un resultado de basculamiento de bloques tectónicos (D umont, 1989), relacionado a la iniciación del plano de subducción bajo los A ndes (Jordan 
et al 1983). Durante estas fases tectónicas muchas de las fallas del Hercyniano fueron reactivadas, principal mente en sentido inverso (L aurent y Pardo, 1975); (L aurent, 1985).

Las presentes áreas de subsidencia de la cuenca del M arañón están caracterizadas por la ocurrencia de grandes áreas inundadas, localizadas de norte a sur, a lo largo del río Pastaza, en la confluencia de los ríos M arañón y Huallaga (L aurent y Pardo 1975) y sobre la parte sur del área de la cuenca M arañón - U cayali, conocida como la depresión U camara (Villarejo, 1988). Esas áreas de activa subsidencia coinciden aproxidamente con deje de la estructura de la cuenca, que parece ser arqueada, tendiendo al N-S en la parte del norte y N W-SE en la del sur (Sanz, 1974; L aurent y Pardo, 1985).

\section{DEPRESION UCAMARA}

La depresión U camara (Fig. 2) es extremadamente plana, drenada por una intrincada red de ríos meándricos y con permanentes o semipermanentes áreas inundadas y lagos (Cabrera La Rosa, 1943; Villarejo, 1988). La depresión es sutilmente delimitada en el norte por el río Marañón (excepto en el bajo Chambira y en el área del río Tigre) y en el oeste por el brazo nortesur del río Samiria, mientras los bordes del sur y del este son definidos por límites morfoestructurales, formados por la falla Tapiche, a lo largo del levantamiento de la Sierra de M oa y la línea de riscos al margen del geoanticlinal Iquitos.

Tres ríos de agua blanca cruzan la depresión, de norte a sur, respectivamente; el M arañón, U cayali y Tapiche; grandes áreas inundadas son localizadas a la largo del curso de los ríos principales que lo cruzan o se localizan adyacentes a los cursos de los nos.

\section{REGIMEN ACTUAL DE LOS RIOS}

El promedio de precipitación anual en la amazonía occidental está sobre los 2,000 m. m., la precipitación cae en todos los meses del año, pero es más alta entre enero y mayo; tal corno ocurre comúnmente en las regiones amazónicas, los drenajes de las tierras bajas están separados formando grandes ríos de agua blanca (agua proveniente de sedimentos de áreas de los A ndes y de las estribaciones) y pequeños ríos de agua negra (agua de precipitaciones rica en ácidos orgánicos que fluyen de las áreas de inundación).

L a diferencia entre el nivel de agua alto (enero a mayo) y el nivel de agua bajo (junio a diciembre) es superior a $11 \mathrm{~m}$. en Iquitos (García y Shna, 1987), pero decrece significativamente hacia el oeste: $9.5 \mathrm{~m}$ en Jenaro Herrera, sobre el borde de la depresión, siendo de menos de $2 \mathrm{~m}$. en la depresión U camara (observación personal). Estas diferencias son debidas probablemente al extraño efecto de las tierras altas de lquitos.

M uy pocos y relativamente imprecisos datos topográficos son disponibles para el área. De acuerdo con las elevaciones de Iquitos (105 m.), N auta (I11 m.) y Requena (114 m.) (M inisterio de Guerra, 1984). La gradiente para los ríos M arañón y U cayali, cruzando el geoanticlinal de Iquitos es de cerca de $0.06 \mathrm{~m} \mathrm{Km.-1} \mathrm{(Fig.3).} \mathrm{Hacia} \mathrm{la}$ cuenca del Marañón, una similiar gradiente media fue establecida entre Requena y Contamana (134 m.) a lo largo del río U cayali. Datos de Stiglish (1904), muestran una gradiente de $0.04 \mathrm{~m}$. K m: 1 entre $\mathrm{M}$ angua (actualmente Ilamado Carolina) y Requena, a lo largo del río U cayali, a través de la depresión U camara; aguas abajo de Iquitos, en 
dirección al mar, el río A mazonas tiene una pendiente promedio de $0.03 \mathrm{~m}$. $\mathrm{Km}$ : 1, el mismo valor es mencionado por Baker (1978) entre M anaus y el mar.

\section{LAS AMPLIAS AREAS DE INUNDACION}

Las permanentes áreas inundadas (Fig. 2) son grandes áreas que no están directamente relacionadas con formas de tierra fluviales; sin embargo, algunas formas de tierra fluviales inundadas semejan meandros, terrenos húmedos o partes de canal que pueden haber sido incorporadas dentro de las más extensivas áreas de inundación. Tres permanentes áreas inundadas han sido identificadas EI área Chambira Tigre (Condordia), en el lado norte del río M arañón, el rea Puinahua, sobre la parte central del canal Puinahua y el área Punga, a lo largo del río Tapiche; todas esas áreas inundadas drenan agua negra. Cuando ellas son cruzadas por ríos de agua blanca, como el área de inundación Punga por el río Tapiche, entonces las aguas blancas y negras están separadas por canales fluviales.

El área de inundación Chambira Tigre (Concordia), está localizada entre el río Chambira hacia el oeste y el río Tigre hacia el este, y se extiende cerca de $25 \mathrm{Km}$. al norte del río $M$ arañón. Esta área está caracterizada más por bosques de tierras húmedas que por áreas inundadas profundas, representada por formas de tierra fluvial inundada, tales como ríos tipo lago.

El área de inundación Puinahua cubre un área de cerca de $25 \mathrm{Km}$. x $60 \mathrm{Km}$., siguiendo el eje del canal Puinahua; hacia el noreste, las permanentes áreas de inundación son discontinuas, con áreas inundadas localizadas alrededor de grandes meandros. Hacia el suroeste, las áreas inundadas son más continuas y las relaciones con las formas de tierras fluviales son obvias. Cuando el límite suroeste es muy marcado y corresponde con una línea de dirección NNW - SSE. La sobre posición de las áreas de inundación sobre las formas de tierra fluvial, sugiere que la primera es posterior a la segunda.

El área de inundación Punga es de forma rectangular $(675 \mathrm{Km} 2)$ y está localizado entre Santa Elena y Wicungo, en ambos lados del río Tapiche $(5 \times 15 \mathrm{Km}$. en el lado oeste yl5 x $40 \mathrm{Km}$ en el este). Stiglish (1904) describió el área como una tierra alta (bosque de altura) y este convincente testimonio apoyado por morfológicas observaciones, sugiere que la inundación de las áreas ha ocurrido hace solamente 60 años. De acuerdo al testimonio de dos habitantes (A rturo Pereira-Iquitos y Santiago Panduro-Santa Elena) el área Punga empieza a hundirse entre 1927 y 1929; después de algunos temblores ocurridos (comunicación personal de Santiago Panduro, el área fue progresivamente inundadas, habiendo tenido colonos e indios Capanahuas que abandonaran cada año las restantes tierras secas que fueron reducidas por inundación. De acuerdo con los datos de los viajeros (F aura, 1964; V illarejo, 1988), la parte baja de los tributarios del río Tapiche 


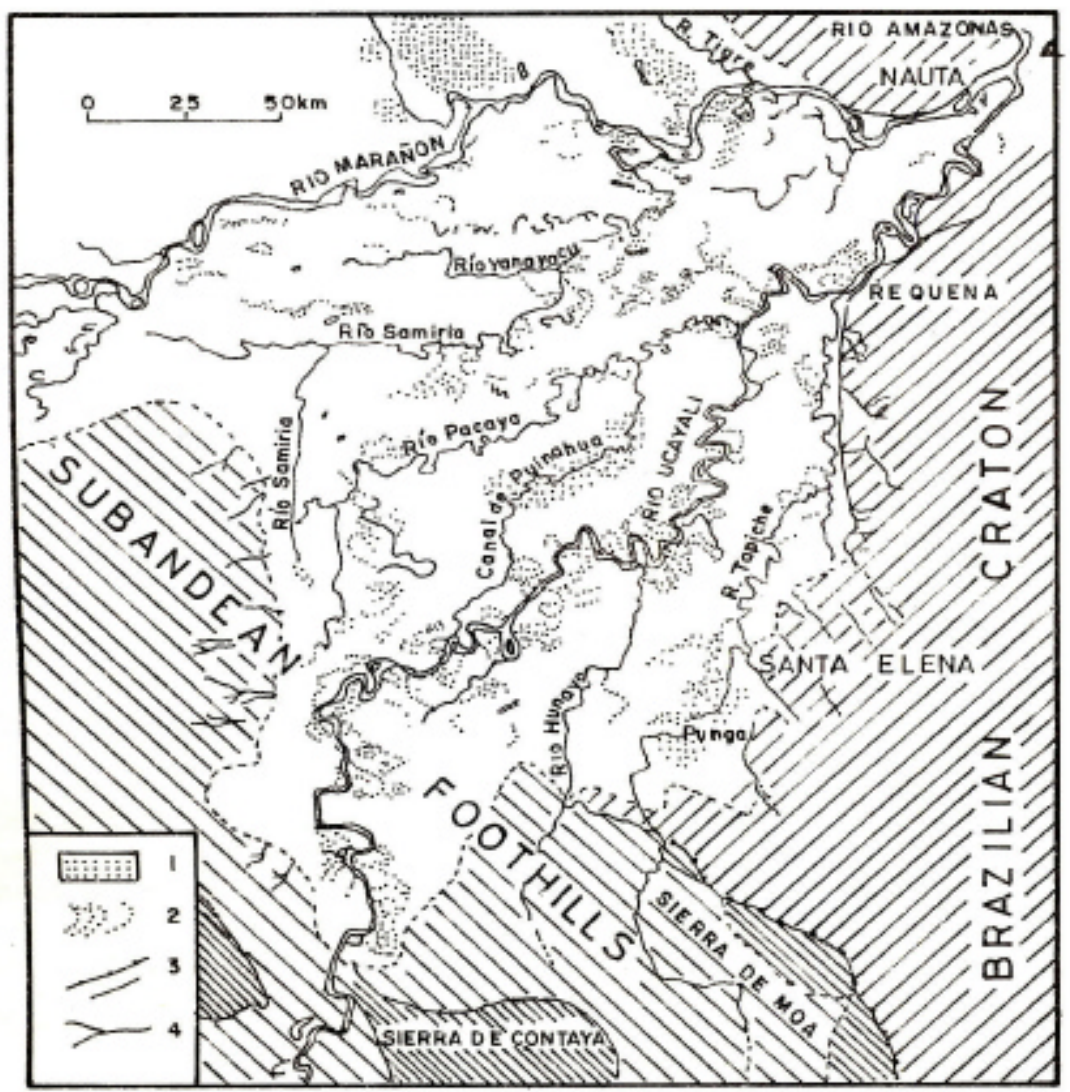

Fig. 2 Esquema morfoestructura de la depresión Ucamara 1.- Mayores áreas de inundación.

2.- Formas de tierra fluviales.

3.- Escarpas morfologicas.

4.- Drenaje en tierras santas

(especialmente el Loboyacu) no fueron completamente inundados entre los años 1930 40. Después de un inicial (relativamente corto) alto porcentaje de inundación, la presente extensión del área fue extendida muy progresivamente. Todo lo que queda de los bosques son troncos de árboles, preservados por las aguas negras y escasamente elevados sobre el bajo nivel de agua. Las bases de los árboles muertos están en el presente bajo $2 \mathrm{~m}$. de agua, durante las bajas crecientes. Estimados basados sobre los presentes bancos de los ríos $(1.5 \mathrm{~m}$. de alto) y el nivel del agua durante las altas crecientes, que sobrepasa el nivel más alto por cerca de $50 \mathrm{~cm}$., sugiere una total subsidencia de más de $4 \mathrm{~m}$.

Se sugiere que la inundación del área Punga está relacionad a la subsidencia tectónica; se desecha la hipótesis de la inundación, debido al elevamiento del nivel de base aguas abajo, porque esto habría inundado no solamente el área Punga, sino también 
la divisoria de agua entre el río Tapiche y el U cayali, que es escasamente más alto que el nivel de agua más alto. A lgunos cambios en la red hidrográfica de la región resultan de la subsidencia del área Punga; los valles de los ríos L oboyacu y Camungo, tributarios orientales del río Tapiche, fueron completamente inundados y el río Tapiche ha migrado al sur hacia el centro del área de subsidencia. El río Tapiche está en el presente reconstruyendo canales sedimentarios a través del área de inundación, por un área levantada de $1 \mathrm{~m}$. a $1.5 \mathrm{~m}$. de alto y menos de $5 \mathrm{~m}$. de ancho, arborizada en algunos sitios por una única hilera de Cecropia (árboles del primer estadio de sucesión forestal, Salo et al, 1986).

\section{EVIDENCIAS DE CONTROL ESTRUCTURAL DEL BASAMENTO}

De acuerdo con Laurent y Pardo (1975), pocas deformaciones son reportadas de los depósitos del Mesozoico y Cenozoico en la cuenca $M$ arañón; pero, por el contrario, el basamento pre-Mesozoico está dividido en varios bloques fallados y levantados. U na comparación entre el esquema de las estructuras del basamento, de un lado, y el esquema de formas de tierra fluviales - grandes áreas de inundación, por otro lado, sugiere que existe un activo control estructural sobre la superficie formas de tierra por el basamento; los más llamativos casos serán reportados aquí (Fig. 3).

El área de inundación Chambira -Tigre está localizada sobre una zona generalmente depresionada en el basamento, zonas del Paleozoico inferior y superior están limitadas por fallas normales, de las cuales una parte fue inversamente reactivada durante las últimas fases tectónicas del Terciaria (Quechua). La parte del oeste del área de inundación del río Chambira cubre un área de horsts (levantamiento Concordia) y gravens profundos, con un basculamiento en general al oeste que termina contra la zona del horsts Patayacu. La rama occidental del levantamiento Concordia ha sido inversamente reactivada durante la última fase tectónica; la parte oriental del área de inundación (río Tigre) cubre (suprayace) en dirección N NW - SSE en profundidad a una zona del Paleozoico inferior limitada por fallas normales. AI norte, las fallas de la zona del este fueron inversamente reactivadas recientemente; de acuerdo con $L$ aurent y Pardo (1975), el efecto de la fase tectónica Quechua sobre la cuenca Marañón fue caracterizado por diferenciales movimiento de los bloques verticales.

El límite de la subsidencia Punga corresponde relativamente con la parte del noroeste del levantamiento Santa Elena, definido en Laurent (1985). La estructura del levantamiento Santa Elena (Alto de Santa Elena) es interpretado como un horsts cristalino rodeado por estratos Paleozoicos; la dirección NNE - SSW del área de inundación Punga es paralelo a algunos de los rasgos estructurales reportados por Laurent (1985), justo hacia el norte y noroeste de la zona elevada de Santa Elena. La posición del área de inundación Punga sugiere que los infrayacientes bloques fallados están aún activos en el basamento. La orientación NNE-SSW del área Punga es sub paralela al sistema en "echelon" (escalera) de la zona de falla del M arañón, reportada para el último Paleozoico (Laurent, 1985). La ubicación de la presente activa 
subsidencia, justo sobre una zona históricamente levantada, sugiere un movimiento recurrente inverso de las fallas, probablemente normales o normal-transcurrentes del Paleozoico.

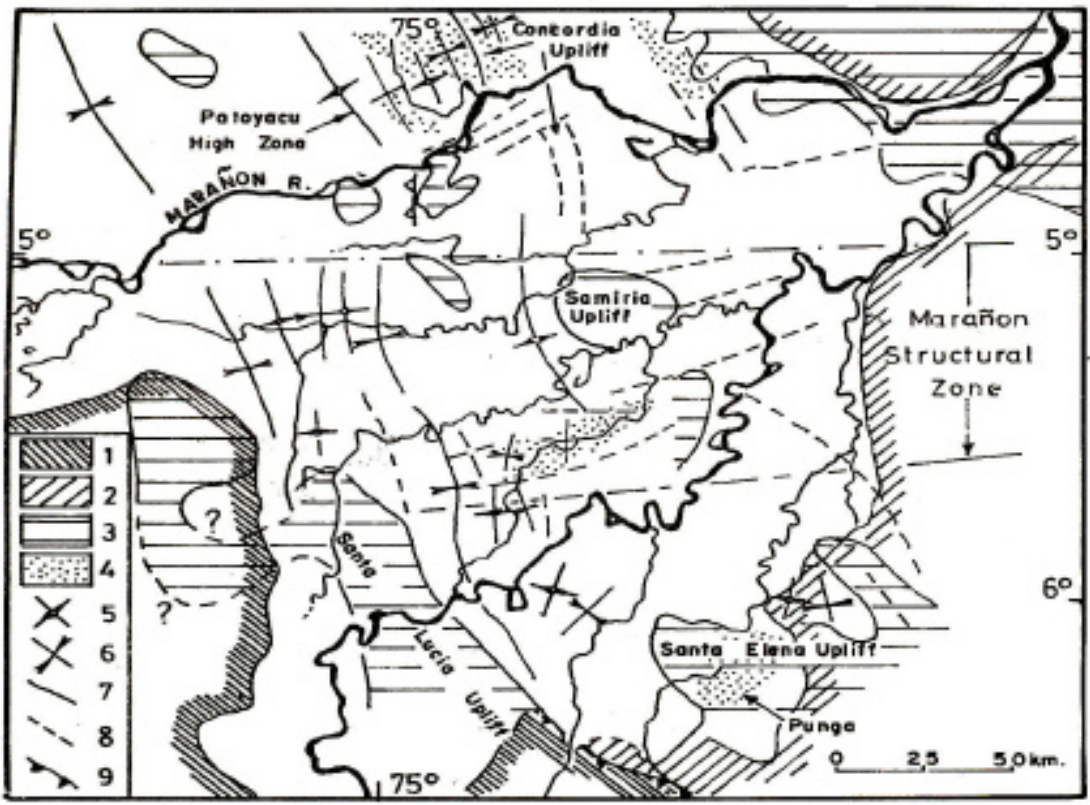

Fig. 3 : Esquema de las estructuras del basamento de la depresión Ucamara Laurent (1985: y Laurent y Pardo (1975), completado con superficie morfoestructurales

1 : Limite de las estribaciones subandinas

2 : Limite de las tierras altas del geoanticlinal lquitos

3 : Ultimos levantamientos Hercynianos de rocas cristalinas sobrecubiertas por depósitos cretáceos

4 : Mayores áreas inundadas

5 : Anticlinales pre-cretáceos

6 : Sinclinales pre-cretáceos

7 Fallas del basamento reactivados durante el útimo tectonismo del terciario

8 : Fallas del basamiento pre-cretáceo

$9 \quad$ : Zona de falla Tapiche con levantamiento post-pleistocénico

El área de inundación Puinahua está localizada dentro de las depresiones del Paleozoico, limitada hacia el norte, este y sur por transcurrentes fallas en el basamento; el límite occidental del área de inundación, que es también el más evidente, es definido por una faja plegada paralela a bordo oriental del levantamiento de Santa Lucia (L aurent, 1985). El caso del área de inundación Puinahua, muestra que la ocurrencia de fallas del Paleozoico en el basamento determina los límites de los bloques subsidentes.

La migración del río U cayali se extiende sobre un área caracterizada por un fallamiento transcurrente N NE -SSW a W-E en el último Paleozoico, relacionado con la zona de falla $M$ arañón; por el contrario, el río $M$ arañón que entra en la cuenca por la zona de falla Marañón, hacia el sur, y la zona de falla Corrientes, hacia el norte. El curso común del U cayali y del Marañón, durante el estadio Samiria, que corresponde al 
límite de la faja de alta densidad de fallas, identificada por L aurent (1985), como la zona de falla: 1 M arañón.

\section{RELACIONES CON LA TECTONICA REGIONAL}

Las geométricas relaciones entre áreas de inundación y estructuras del basamento no son las mismas por toda la depresión; en el norte de la zona de falla del $M$ arañón, áreas de inundación y estructuras del basamento son paralelas a las principales estructuras andinas (NNW -SSE). En la parte sur de la depresión, la dirección de elongación del área de inundación parece ser controlada por estructuras transversales NE - SW a NNE - 55W, relacionada a la zona de falla M arañón.

$L$ a más activa subsidencia de la cuenca $M$ arañón ocurre en el presente enfrente del área de escurrimiento subandino y cinturón de pliegues (STFB), en la parte norte de la cuenca y se extiende hacia el sur, enfrente de la parte norte de la falla inversa Tapiche; esto puede ser interpretado como una tendencia de la presente cuenca a extenderse hacia el sudeste, 0 a lo largo del eje Cretáceo preexistente de la cuenca, que se extiende sobre las áreas Pastaza, U camara - A cre.

El estado de tensión en la depresión U camara es desconocido, pero el caso del área de inundación Punga sugiert que el basamento está sometido a esfuerzos de comprensión. En opinión de los autores, esta compresión es más débil que en el Perú Central, donde el total del frente subandino y el borde del cratón están sometidos a levantamientos. La combinación de un buen ajuste compresional en el Perú central y uno débil en el norte del Perú, puede producir algunos ajustes en el borde del cratón, semejantes al fallamiento normal observado en las tierras al tas de Iquitos (D umont et al, 1988). Esas fallas, de relativamente corto empuje, tienen un mayor control sobre los límites de la llanura de inundación, lo cual puede ser interpretado como generación de junturas neotectónicas superficiales de pequeña escala (Hancock y Engelder, 1989). EI fallamiento tectónico es contemporáneo con el elevamiento de las tierras altas de Iquitos durante el Cuaternario; el incremento de la tectónica conduce a una más activa subsidencia del cratón (efecto rebote), dando como resultado un embalse y originando el funcionamiento de todos los drenajes, comprendiendo la cuenca $M$ arañón, hacia solamente una salida, dando origen al río A mazonas.

\section{CONCLUSIONES}

En la activamente subsidente depresión U camara, las relaciones entre las grandes áreas inundadas y el basamento estructural son obvias; algunos casos, están claramente relacionados con estructuras del Hercyniano y fallas inversamente reactivadas durante las últimas fases tectónicas del Terciaria y Cuaternario; otros casos son menos evidentes, pero mas probablemente relacionados a la reactivación de estructuras inversas o trascurrentes de estructuras del basamento. 


\section{BIBLIOGRAFIA}

ALLEBY, R.J. 1988. Origin of rectangular and aligned in the Beni Basin of Bolivia. Tectonophysic 145, 1-20.

BAKER,V.E. 1978. Adjustment of fluvial system of climate and source terrain in tropical and subtropical environments. Ing: Fluvial Sedimentology, A.D. M iall, (Editor), Can Soc. Pet. Geol., Mem. no 5,211-230.

CABRERA LA ROSA, A. 1943. Características geomorfológicas de los ríos en la región A mazónica. Bol. Soc. Geol. Perú 14 \& 15, 38-58

DUM ONT,J.F. 1988. Neotectónica y dinámica fluvial de la baja Amazonía Peruana Bol. Soc. Geol. Perú 80 51-64

DUMONTKJ.F; S. LAMOTTE \& M. FOURNIER (1988). Neotectónica del Arco de Iquitos (J enaro Herrera, Perú). BoL Soc. Geol. Perú 77. 7-17/

FAURA, G.S. 1964. Los ríos de la A mazonía peruana. Ed. Colegio Militar Leoncio Prado (Callao, Perú)

GARCIA.JS. \& SERVICIO DE HIDROGRAFIA Y NAVEGACION DE LA AMAZONIA 1987. El Río que se aleja: Cambio del curso del Amazonas, historia y estudio técnico. Centro de Estudios Teológicos de la Amazonía, Iquitos.

HAM , C.K. \& U. HERRERA 1963. Role of Subandean fault system in tectonics of Eastern Peru, and Ecuador. In: O.E. Childs and B.W. Beebe (Editors), Backbone of the A mericans. Am. A ssoc. Pet. Geol., Mem. № 2,47-61.

HANCOCK, P.J. \&T. ENGELDER 1989. Neotectonic joints. Bull Geol. Soc. Am. 101,1197- 1208.

IRIODON, M.\& K. SUGUIO 1981. Neotectonies of the Amazon plain INQUA N eotec. Bull. 4,72-78.

JORDAN, T.E.; B.L. ISACKS; R.W. ALLMENDINGER; J.A. BREWER; V.A. RAMOS \& Cj. ANDO 1983. Andean tectonics related to geometry of subducted Nazca plate. Bull Geol. Soc. A m. 94,341-361.

LAURENT, H. 1985. El pre-Cretáceo en el oriente peruano: su distribución sus rasgos estructurales. Bol. Soc. Geol. Perú 45,25-48 
LAURENT, H. \& A. PARDO (1975). Ensayo de interpretación del basamento del Nororiente Peruano. Bol. Soc.Perú45, 25048.

MEGARD, F. (1984). The A ndean orogenic period and its mayor structures in central and northern Peru J. Geol. Soc. London 1411, 893-900

MINISTERIO DE GUERRA (1984). M apa físico-político del Perú.

PARD O, A. (1982). Características estructurales de la faja subandina del norte del Perú In Symposium "Exploración Petrolera en las Cuencas Subandinas de Venezuela, Colombia, Ecuador y Perú ", A soc. Colomb. Geol. Geofis. Petr, Bogot.

RUEGG, W. (1952). La depresión del U cayali y A mazonas superior Rey. A ssoc. Geol A rgentina 7, 106-124.

SALO,J; R. KALLIOLA; 1. HAKKINEN; 1. MAKINEN; P. NIEMELA; M. PUHAKKA \& D. COLEY (1986). River dynamics and the diversity of A mazon lowlands forest. Nature, 322, 254-258.

SANZ, V.P. (1974). Geología preliminar del Río Tigre-Corrientes en el Nor oriente peruano. Bol. Soc. Geol Perú 44, 106-127.

STERNBERG, H.O R. (1950). Vales tectonicas na planicie amazónica Rey. Bras. Geogr. 4(12), 511-534.

STERNBERG, Hø.R. (1955). Sismicit et morphologie en A mazonie brcilienne. A nnales de Geographic 342,97-105.

STIGLISH, G. (1904). Ultimas exploraciones ordenadas por la Junta de vías fluviales a Ios ríos U cayali, M adre de Dios, Paucartambo y U rubamba Oficina tipográfica de "L a O pinión N acional".

VILLAREJO, A. (1988) (first publication 1943) Así es la Selva, Centro de Estudios Teológicos de la A mazonía, Iquitos. 